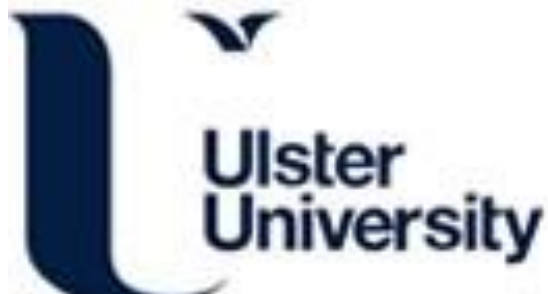

\section{The Application of Social Media Image Analysis to an Emergency Management System}

Jing, M., Bryan, S., Coleman, SA., \& McGinnity, TM. (2016). The Application of Social Media Image Analysis to an Emergency Management System. In Unknown Host Publication (pp. 805-810). IEEE. https://doi.org/10.1109/ARES.2016.24

Link to publication record in Ulster University Research Portal

\author{
Published in: \\ Unknown Host Publication
}

Publication Status:

Published online: 15/12/2016

DOI:

10.1109/ARES.2016.24

\section{Document Version}

Author Accepted version

\section{General rights}

Copyright for the publications made accessible via Ulster University's Research Portal is retained by the author(s) and / or other copyright owners and it is a condition of accessing these publications that users recognise and abide by the legal requirements associated with these rights.

\section{Take down policy}

The Research Portal is Ulster University's institutional repository that provides access to Ulster's research outputs. Every effort has been made to ensure that content in the Research Portal does not infringe any person's rights, or applicable UK laws. If you discover content in the Research Portal that you believe breaches copyright or violates any law, please contact pure-support@ulster.ac.uk. 


\section{The Application of Social Media Image Analysis to an Emergency Management System}

$\begin{array}{cc}\text { Author } & \text { Author } \\ \text { Address } & \text { Address } \\ \text { emails } & \text { email }\end{array}$

\begin{abstract}
The emergence of social media has provided vast amounts of information that is potentially valuable for emergency management. In the EU-FP7 Project Security Systems for Language and Image Analysis (Slandail), an image analysis system has been developed to recognize the flood water images from the social media resources by incorporating with text analysis. A novel image feature descriptor has been developed to facilitate fast image processing based on incorporation of the "Squiral" (Square-Spiral) Image Processing (SIP) framework with the "Speeded-up Robust Features" (SURF). A new approach is proposed to generate an index from image recognition outcomes based on a moving window average, which presents a temporal change based on the occurrence of flooding water identified by image analysis. The evaluation for computation time and recognition were based on a batch of images obtained from the US Federal Emergency Management Agency (FEMA) media library and Facebook corpus from Germany, and the outcomes show the advantages of the proposed image features. The simulation results demonstrate the concept of the index based on a moving window average, highlighting the potential for application in emergency management.
\end{abstract}

Keywords-flood event image recognition; fast image processing; social media analysis; emergency management.

\section{INTRODUCTION}

Social media comprise contextual information such as tags, comments, geo-locations and metadata arising from the capture device, which are valuable for webbased applications. The use of social media in disaster and crisis management is increasing within the EU. In recent EU-FP7 Project Security Systems for Language and Image Analysis (Slandail) [10], the end-user partners, An Garda Siochana (Irish Police), Police Service of Northern Ireland, Protezione Civile Veneto, and Bundeskommando in Leipzig Germany, have reported use of social media together with legacy media for natural disasters focusing on flooding events in Dublin, Belfast, Venice and Leipzig, respectively. Techniques developed for visual content analysis are valuable for improving search quality and recognition capabilities of current emergency management systems. A recent study [4] has shown that whilst the current focus in disaster management systems is on text analytics, visual content made available through social media will initially leverage text analytics and in the longer term image analytics will have a profound positive impact on disaster management. Attention has been focused on fusing textual and visual aspects. For example, flood water image recognition can be enhanced by incorporating text analysis at the feature level [5]. The texts linked to the image captions and titles have been used together with image features for categorizing images [1]. Machine learning and neural network systems have also been used to train systems to automatically annotate images with keywords found in collateral texts [13].

Fast image processing is a key element in achieving real-time image and video analysis, which is a challenging task, particularly when handling large-scale image and video data from social media. A recent work has developed a novel "Squiral" (Square-Spiral) Image Processing (SIP) framework that introduces a spiral 1-dimensional addressing scheme for standard square pixel-based images [3]. The SIP-based approach enables the image pixel values to be stored in a 1D vector, facilitating fast access and accelerating the execution of subsequent image processing algorithms by mimicking aspects of the eye tremor phenomenon in the human visual system [9]. Conversion of standard two-dimensional pixel indices to the 1D SIP addressing scheme can be achieved easily using an existing lattice with a Cartesian coordinate system. Furthermore, the approach can be used for efficient convolution of existing image processing operators designed for standard rectangular pixel-based images and does not require any new operators to be developed. This work introduces a new image feature descriptor developed based on an extension of the SIP framework.

This paper also presents the process of integration of the image recognition system developed to a flood event management system, including to develop a proper output format for system data exchange and to generate a meaningful index for the end users. Flood event forecasting and monitoring provide vital information for emergency management systems. Many existing flood forecasting systems focus on the analysis of large-scale areas based on satellite, optical, and radar images [7], [11], [12]. These forecasting systems subsequently provide information to support identification of flood areas and estimation of flood levels. However such methods based on satellites or radar images may not be available to emergency management systems. Additionally the traditional media system can be affected by adverse weather conditions during flood events. Social media has the advantages over satellite radar image based analysis because it is faster than traditional media and the first-hand information is shared by large audiences from the flood affected area. In this work we develop and integrate the image recognition system to identify the flood water images obtained from social media 
corpus. Furthermore we propose an approach to generate a flexible and easy interpretable index for the end users based on the outcome from image recognition system.

The remainder of the paper is structured as follows. In Section II the image recognition model is introduced and a novel image feature based on incorporating the SIP framework with SURF is presented. The process of applying the image analysis to disaster management system is described and the approach of generating an index for end users is explained. In Section III, the evaluation of the recognition system performance based on run times and features is provided, followed by the simulation test for index. Conclusions are provided in Section IV.

\section{Methods}

\section{A. Image Recognition via BoW Model}

The proposed image analysis system works on web image resources together with text analysis. The text analysis is performed first to obtain the flood event corpus from a range of resources such as news feeds, government agency web sites and social networking sites. The corpus includes information on event location, time, article titles, descriptions and URLs for images. The URLs are used to extract the flood event images which may contain flood water, people, roads, cars, and other entities. The task for image recognition is to identify the flood water images based on the Bag-of-Words (BoW) model [8]. As shown in Figure 1, the images collected are used for training the recognition system, which includes image feature extraction, learning of visual words and construction of feature representation based on the BoW model. The local image features are first mapped to a codebook created by a clustering method such as k-means and then represented by a histogram of the visual words that is used for classification.

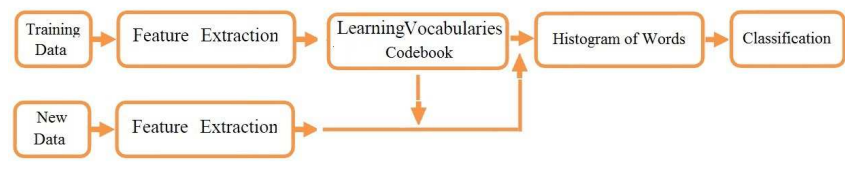

Figure 1: The image recognition system based on the BoW model.

For the image recognition system, the "word" refers to the "visual word", which is represented by a set of feature centres resulting from the clustering method. The classification is based on a Support Vector Machine (SVM). The image analysis outputs are saved in a XML format to enable the further integration of image analysis to a disaster management system.

\section{B. Development of SIP-based Features (SIPF)}

To accelerate fast image processing for social media image analysis, we introduced a new image feature descriptor which incorporates the SIP framework with SURF [2]. According to the SIP framework, layer-1 of the SIP addressing scheme comprises 9 pixels in a spiral pattern as shown at the centre of Fig. 2. Subsequent layers of the SIP addressing scheme are built recursively. The converted SIP image is stored in a one-dimensional vector according to the spiral addresses.

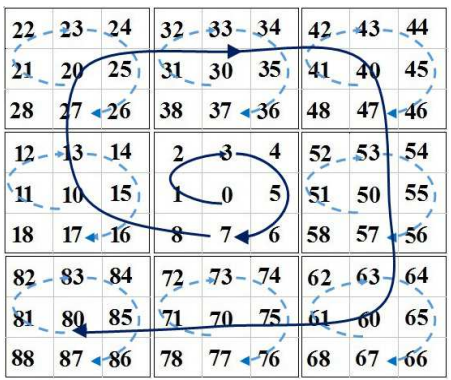

Figure 2: The spiral addressing scheme for layer-2 SIP
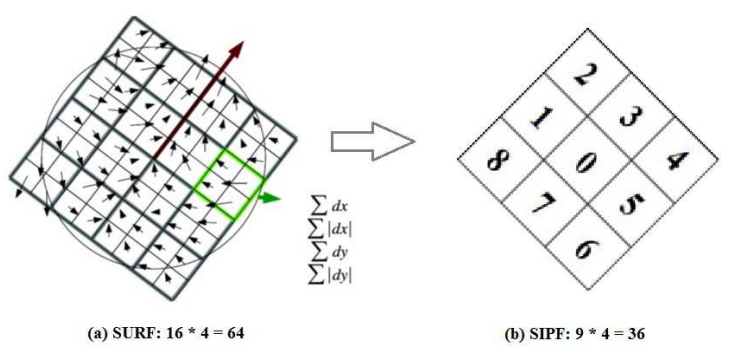

Figure 3: (a) SURF feature construction [2]; (b) SIPF feature based on layer-1 SIP addressing scheme

We incorporate the SIP addressing scheme with the image feature SURF to improve the efficiency of web image analysis. SURF has been used widely in image analysis and has shown advantages over SIFT [6]. It has been demonstrated in [3] that SIP-based convolution produces exactly the same results as standard convolution, and hence in our current implementation we use the interest points detected by SURF but rearrange the SURF features according to the SIP addressing scheme. As shown in Figure 3 (a), the SURF features are constructed based on a square region centred on the detected SURF interest point. The region is divided into smaller $4 \times 4$ sub-regions, and within each sub-region the wavelet responses are computed. The responses include the sums of $d x,|d x|, d y$, and $|d y|$, computed relative to the orientation of the grid, where $d x$ and $d y$ are the Haar wavelet responses in the horizontal and vertical direction respectively; $|d x|$ and $|d y|$ are the sums of the absolute values of the responses, respectively. Hence each sub-region has a four-dimensional descriptor vector [ $d x, d y,|d x|,|d y|]$. Concatenating these for all $4 \times 4$ sub-regions results in a SURF descriptor vector of length 64.

To construct the equivalent using the SIP framework, we apply the layer-1 SIP addressing scheme to rearrange the SURF feature obtained from each interest point. In order to match the layer- 1 SIP structure, the $4 \times 4$ sub-regions are resized to $3 \times 3$ sub-regions using a bicubic interpolation method (in which the output pixel value is a weighted average of pixels in the nearest 4-by-4 neighborhood), 


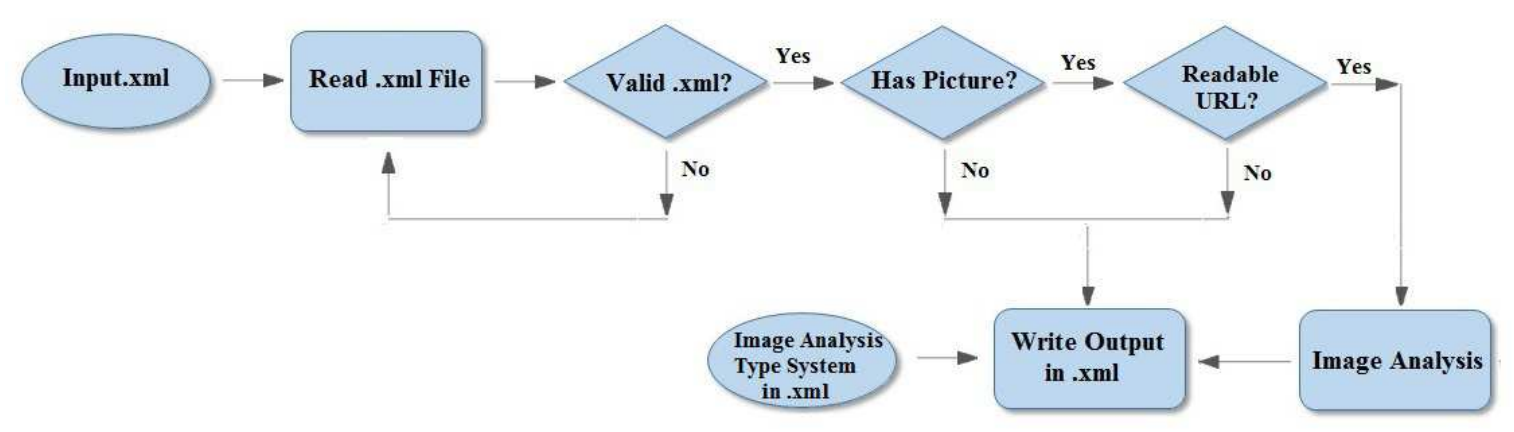

Figure 4: The flowchart of data exchange of image analysis with emergency management platform

and then the corresponding response values are rearranged according to the layer-1 SIP addressing scheme as shown in Figure 3 (b). This results in a descriptor of length $9 \times 4=36$. Note that the current implementation does not involve full SIP image conversion and SIP convolution, but it yields the promising results and therefore preliminary work is encouraging for future development of a full SIP image feature detection algorithm.

\section{Integrating Image Analysis into an Emergency Man- agement Platform}

1) Data Exchange and Flowchart: The Slandail system data exchange uses the XML data format. XMI derived from UIMA CAS objects was chosen for the final emergency management system because of its flexibility in which the data structure (expressed as Type System) can be defined according to the data types and outputs from either text or images analysis. For image analysis the output Type System includes the features of Image Class, Probability Score and Image Feature Histogram, which can be written in XML format once the image analysis Type System is defined. The flowchart of linking the image analysis framework to the emergency management system is shown in Fig. 4, which shows the data exchange between image analysis and the management system.

2) Development of Index: Since the emergency management system is designed for the end users, a key aspect of such an emergency management system is to provide a meaningful index that can be easily interpreted by the end users. Image recognition system based on a binary classifier can provide a classification score, ideally a positive score suggests a flood water image and a negative score indicates a non-flood-water image. However, such scores may not be straightforward for the end users to interpret. In addition the classification scores are returned for each image (at single time point) which does not reflect changes over time. Furthermore, unlike the traditional systems based on satellite images which rely on history data (as reference) for flood monitoring or detection, an emergency management system based on social media analysis may not have such reference data available.

To tackle these problems, we propose an approach to generate a single index from image recognition scores based on a moving window average. By calculating the average of classification scores within a given window frame, the system can reduce the impact of individual scores and reflect the overall change over the defined time window. Mathematically, the moving window average can be achieved by convolution of the scores with a user defined window. We will demonstrate this idea via simulation in Section III.

Apart from using the classification scores to build an index, we further derive a posterior probability score from image recognition output as an alternative option for building the index. An example of the probability tree based on a binary classification system is shown in a diagram in Fig. 5. As seen in Fig. 5, for a binary

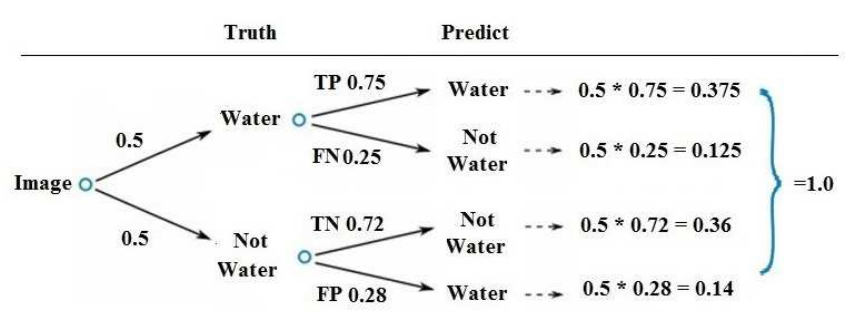

Figure 5: An example of the probability tree derived based on the flood image recognition system. TP: true positive; FP: negative positive; TN: true negative and $\mathrm{FN}$ : false negative.

classification system, the probabilities of an image is recognized as a flood water or non-flood-water are equal, i.e., $\mathrm{P}($ score $>0)$ and $\mathrm{P}($ score $<0)$ are 0.5 . The probability of a flood water image is correctly identified can be found based on the recognition True Positivity (TP). Likewise, the probability of a flood water image is wrongly classified as not-water can be found by False Negative (FN). The posterior probability of an image identified as a flood water image when score $>0$, such as $\mathrm{P}(\mathrm{C}=$ Water $\mid$ Score $>0)$ can be calculated based on conditional probability, which is

$$
\frac{P(\text { Score }>0) \times \text { Likelihood }(\text { Score }>0 \mid C=\text { Water })}{P(C=\text { Water })}
$$

The likelihood of (Score $>0 \mid \mathrm{C}=$ Water) is the probability of score $>0$ when an image is identified as water, which can be found by the true positive (TP) rate. The posterior probability score for $\mathrm{P}(\mathrm{C}=$ Water $\mid$ Score $>0)$ is equivalent to calculate the recognition Precision, where Precision $=$ $T P /(T P+F P)$ and can be obtained from the system 
training.

\section{EXPERIMENTAL RESULTS}

\section{A. Data}

The flood event corpuses were collected from two sources, the US FEMA media library and public Facebook pages and groups (in German) related to flood and flood aid which represent the resources of a government agency and a social networking site respectively. The images were extracted from the web sites using a web scraping tool. Each source has images with different levels of quality in terms of image size and resolution, which is used for comparison purposes. The FEMA images are obtained in their original size with dimensions of 2000-4000 pixels. The Facebook images size are smaller than FEMA, which have a maximum height of 720 pixels. The images were selected and categorized into two groups: flood water and background (the background images does not contain flood water). The focus of this work is to distinguish the flood water images from the background images. In the BoW model, the number of words used was 500 and the recognition performance was evaluated based on Average Precision (AP).

\section{B. Evaluation based on Batched Images}

To test the speed of image recognition, run time based on the analysis of a batch of images was recorded. The system was trained first and the parameters were saved for testing. The training time is not included in the run time. A total of 500 images were used for testing (250 images taken from group). Each run includes: read image from a given path, image pre-processing (standardize the image to have maximum height of 480 pixels and convert the colour images to grey index images), image feature extraction, calculation of histogram of the image features and SVM classification. The image feature used are SIFT, SURF and SIPF.

1) Run Times: The processing run time was recorded and results based on FEMA and Facebook data are given in Fig. 6 and Fig. 7 respectively. For both FEMA and Facebook data, it can be seen that SIPF and SURF are faster than SIFT. The times for SIPF and SURF are comparable for Facebook data. For FEMA data, SURF is slightly faster than SIPF due to the time taken to remap to the SIPF framework from SURF. However, SIPF is faster than SURF if this remapping time is excluded as reported in [4]. In terms of data, because FEMA images are larger than Facebook images, it take longer time to process them.

2) Recognition: We further compare the recognition AP based on SIPF and SURF. The results based on FEMA and Facebook data are shown in Fig. 8 and Fig. 9. It can be seen that for both datasets, SIPF performs better than SURF. In addition, despite the difference in image quality, i.e. FEMA images are in their original form whereas Facebook images are from social media, SIPF works well for both data, which demonstrate the advantages of the proposed new feature SIPF.

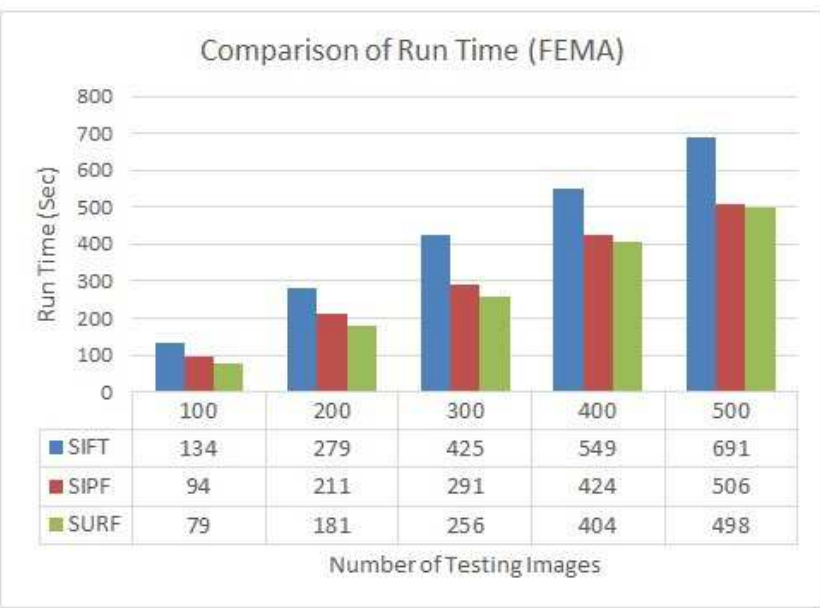

Figure 6: Comparison of Run Time based on FEMA Images using SIFT, SURF and SIPF.

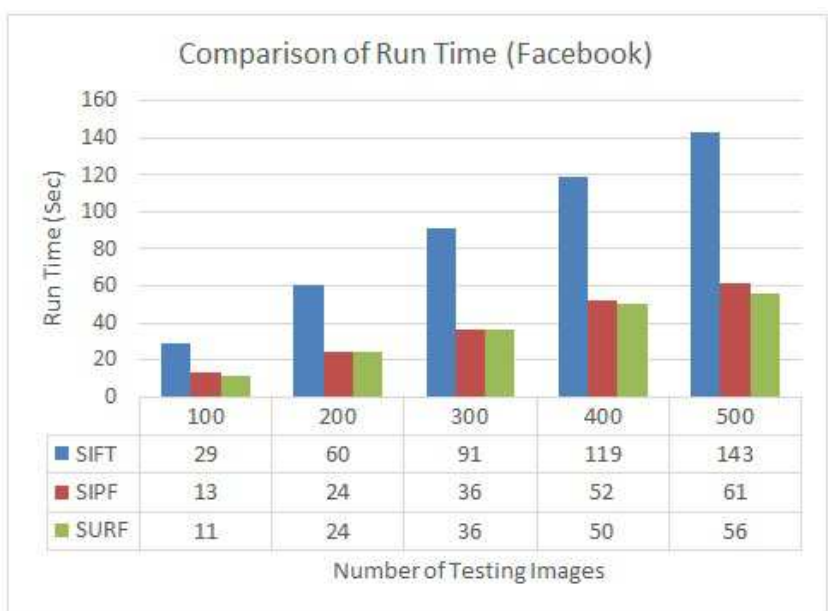

Figure 7: Comparison of Run Time based on Facebook Images using SIFT, SURF and SIPF.

\section{Simulation Test for Index}

In this experiment we set up a simulation to demonstrate the proposed method based on a moving window average to generate a meaningful index from flood image recognition. Before testing, the system was trained based on 600 Facebook images including 300 for each of flood water and background group. Five-fold validation was performed and the recognition rates for TP, FP, TN and FN were calculated. A total of 100 Facebook images are used for testing, which include 50 flood water images and 50 background images. We consider the input images as a time series. The first period is assumed as the normal condition that contains 50 background images mixed with several flooding water images at the beginning. The second period is considered as the flood event period, which contains all flooding water images. Image analysis was performed on 100 images, the classification scores obtained and probability scores calculated (as explained in Section II.C.2).

The outcomes of image recognition is given in Fig. 10, which shows the top 36 flood water images ranked by the classification scores. The classification scores together 


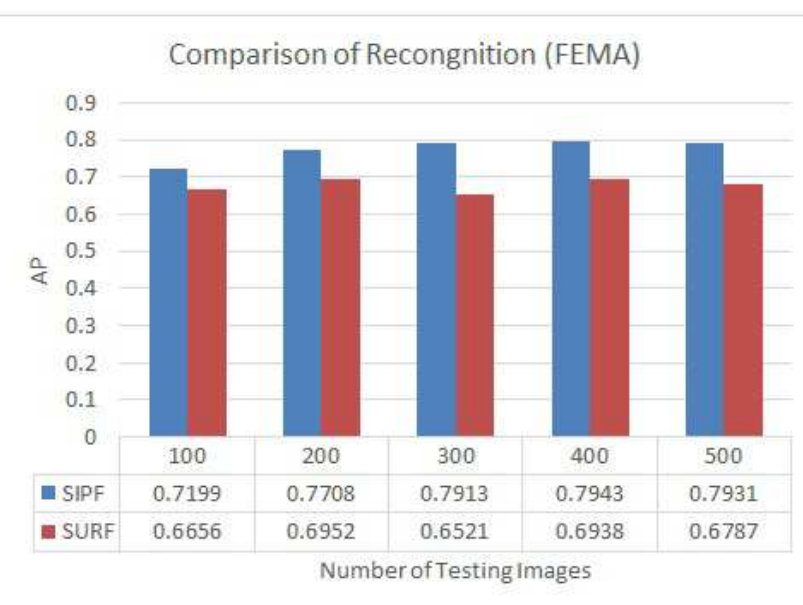

Figure 8: Comparison of Recognition based on FEMA Images using SURF and SIPF.

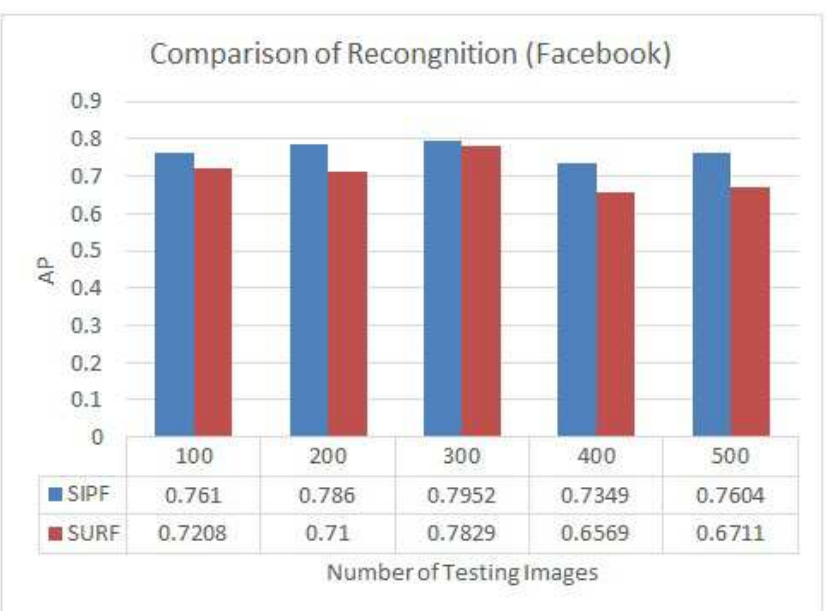

Figure 9: Comparison of Recognition based on Facebook Images using SURF and SIPF.

together with their corresponding moving window average are provided in Fig. 11. The figure shows that the scores change according to the input, such as a positive score indicates the flood water image and a negative score suggests the background image. The index based on the moving average gives a smooth version of the changes. Some high classification scores appear at early in the series because the system identifies several flood water images. However, the index remains low until the second period (after the flood event occurs). The results based on probability and its moving average is given in Fig. 12, which indicates a similar trend as in Fig. 11. The results demonstrate the advantages of using moving averages, which mitigates the effect of individual scores and reflects the overall changes in a given time window. In addition, this index is flexible as the end users can set up the window width according to their needs.

\section{CONCLUSION}

The work presents the integration of an image analysis system to an emergency management system based on social media data analysis. A novel image feature descriptor

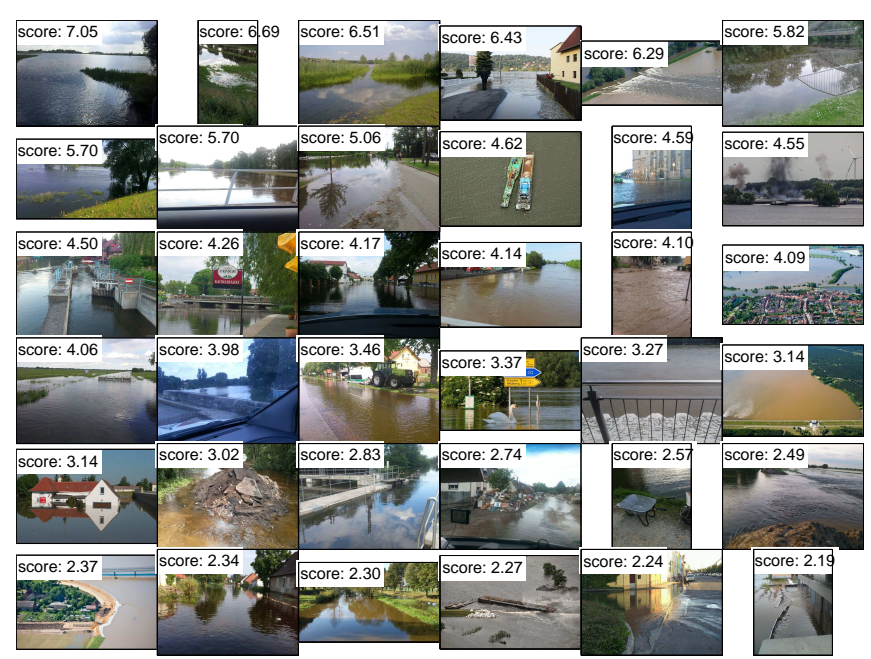

Figure 10: Facebook image recognition shows the top 36 flood water images ranked by the classification scores.

based on incorporating the SIP framework with SURF for fast image processing has been introduced and the advantages demonstrated by FEMA and Facebook image data. An approach based on a moving window average has been proposed to generate an index from image analysis which can provide a flexible and meaningful index for the end users. In the future work, the index may be improved by adding a weight factor to be further derived from the image recognition. The simulation results suggest the potential of the generated index in applications for emergency management.

\section{ACKNOWLEDGMENT}

The research leading to these results has received funding from the European community's Seventh Framework Programme under grant agreement No. 607691, SLANDAIL (Security System for Language and Image Analysis). The authors also like to thank the project partners from Trinity College Dublin in Ireland and University of Leipzig in Germany to provide the valuable FEMA data and Facebook data.

\section{REFERENCES}

[1] K. Ahmad, M. Tariq, B. Vrusias, and C. Handy, "CorpusBased Thesaurus Construction for Image Retrieval in Specialist Domains," In (Ed). Fabrizio Sebastiani. Proc 25th European Conf on Inf. Retrieval Research (ECIR03) LNCS-2633, Heidelberg:Springer Verlag, pp. 502-510, 2003.

[2] H. Bay, T. Tuytelaars, and L. Van Gool, "Surf: Speeded up robust features”, In Proc. ECCV, 2006, vol. 1, pp. 404-417.

[3] M. Jing, B. W. Scotney, S. A. Coleman, and T. M. McGinnity, "Biologically Inspired Spiral Image Processing for Square Images", In Proc. IAPR MVA, 2015, pp. 102-105.

[4] M. Jing, B. W. Scotney and S. A. Coleman et. al, "Flood Event Image Recognition via Social Media Image and Text Analysis," IARIA conference COGNITIVE, 2016.

[5] M. Jing, B. W. Scotney and S. A. Coleman et. al, "Integration of Text and Image Analysis for Flood Event Image Recognition,”. Accepted by IEEE ISSC, 2016. 


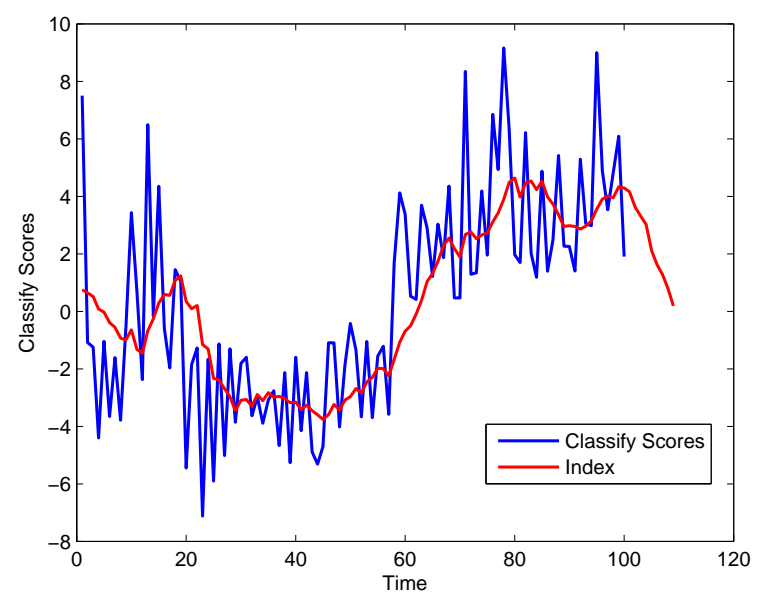

Figure 11: Flood image classification scores and the index generated by moving average.

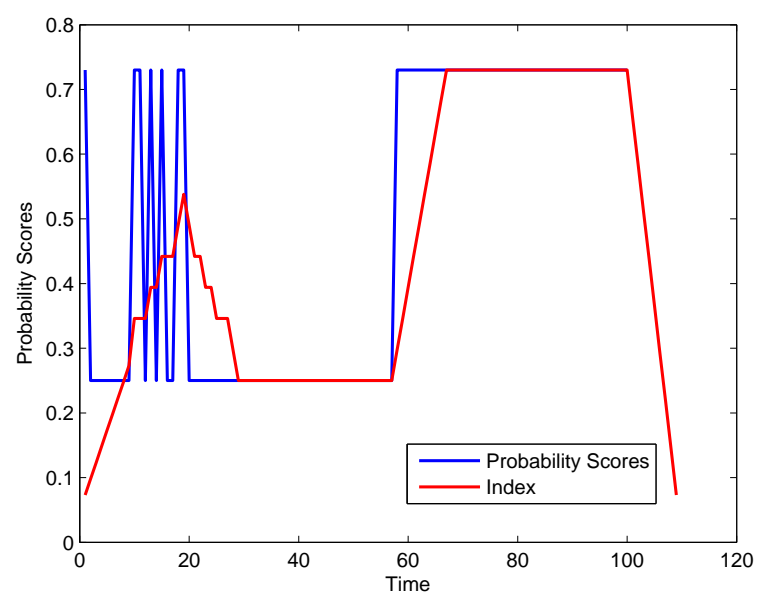

Figure 12: Flood image classification probability scores and the index generated by moving average.

[6] D. G. Lowe, "Distinctive Image Features from ScaleInvariant Keypoints," International Journal of Computer Vision, 2004, vol. 60(2), pp. 91-110.

[7] M. Neiza, L. Nardy, S. Campo, J. Augusto, and E. Posada, "Digital Techniques of Images to Support the Analysis of Flood Hazard," Advances in Remote Sensing, Finite Differences and Information Security, 2012, pp. 115-120.

[8] J. C. Niebles, H, Wang, and L. Fei-Fei, "Unsupervised learning of human action categories using spatial-temporal words," In Proc. BMVC, 2006, vol. 3, pp. 1249-1258.

[9] B. W. Scotney, S. A. Coleman, and B. Gardiner, "Biologically Motivated Feature Extraction Using the Spiral Architecture", In Proc. IEEE ICIP, 2011, pp. 221-224.

[10] FP7 Project Slandail web site: www.slandail.eu.

[11] G. J-P. Schumann. "Editorial: Preface: Remote Sensing in Flood Monitoring and Management," Remote Sens. 2015, 7(12), pp. 17013-17015. doi:10.3390/rs71215871.

[12] S. Voigt, T. Kemper, T. Riedlinger, R. Kiefl, K. Scholte, and H. Mehl, "Satellite Image Analysis for Disaster and CrisisManagement Support," IEEE Transactions on Geoscience and Remote Sensing, 2007, 45(6), pp. 1520-1528.
[13] C. Zheng, A. Long, Y. Volkov, A. Davies and K. Ahmad,"A Cross-Modal System for Cell Migration Image Annotation and Retrieval", 20th IJCNN:International Joint Conference on Neural Networks, pp. 1738-1743, 2007. 